Research Article

\title{
Massage Therapy of the Back Using a Real-Time Haptic-Enhanced Telerehabilitation System
}

\author{
Cristina Ramírez-Fernández, ${ }^{1}$ Victoria Meza-Kubo, ${ }^{2}$ Eloísa García-Canseco, ${ }^{2}$ \\ Alberto L. Morán, ${ }^{2}$ Oliver Pabloff, ${ }^{2}$ David Bonilla, ${ }^{2}$ and Nirvana Green ${ }^{2}$ \\ ${ }^{1}$ Instituto Tecnológico de Ensenada, Ensenada, BC, Mexico \\ ${ }^{2}$ Facultad de Ciencias, Universidad Autónoma de Baja California, Ensenada, BC, Mexico \\ Correspondence should be addressed to Cristina Ramírez-Fernández; cramirezf@gmail.com
}

Received 29 July 2017; Accepted 15 November 2017; Published 31 December 2017

Academic Editor: Pino Caballero-Gil

Copyright (c) 2017 Cristina Ramírez-Fernández et al. This is an open access article distributed under the Creative Commons Attribution License, which permits unrestricted use, distribution, and reproduction in any medium, provided the original work is properly cited.

We present the usability evaluation of a haptic-enhanced telerehabilitation system for massage therapy of the back using the Vybe haptic gaming pad and the gesture sensor LEAP motion controller. The evaluated system includes features that allow for (i) administering online therapy programs, (ii) providing self-adjustable and safety treatment of back massages using a virtual environment, and (iii) saving and replaying massage sessions according to a patient's therapy program. The usability evaluation with 25 older adults and 10 specialists suggests that the haptic telerehabilitation system is perceived with high usability and pleasurable user experience, while providing personalized intensity of haptic therapy in a supervised, real-time, and secure way to treat the patient. Moreover, the specialists totally agree that the system design features, such as save and play, and delimiting therapy zones are the most important for back massage therapy, while the features of regulating feedback intensity and providing/receiving a massage remotely are also important. Finally, based on their comments, five design insights aiming at improving the current version of the system were generated.

\section{Introduction}

Due to a combination of demographic changes and the lack of resources in the field of public health and technology improvement, the development of new rehabilitative practices seems mandatory to build sustainable models for rehabilitation from the clinical, organizational, and economic perspectives [1]. In recent years, haptic feedback has proven to enhance user experience in telerehabilitation [2]. However, current telerehabilitation systems have yet to exploit the richness of haptic modality within its content. The lack of haptic methods that provide real-time rehabilitation in a supervised and remote way inhibits the attention of patients that require massage treatment of the back. Massage therapy has become one of the most popular complementary and alternative medical (CAM) therapies for back pain, the condition for which CAM therapies are most commonly used [3]. A massage is defined as "a mechanical manipulation of body tissues with rhythmical pressure and stroking for the purpose of promoting health and wellbeing" [4]. Despite the availability of novel mechanical support tools for back massage intervention, for example, [5-7], massage therapy is still provided in a traditional way, where both the patient and the therapist need to be in the same place [8].

In [9], we introduced a novel real-time haptic-enhanced telerehabilitation system for massage therapy that consists of a web application and a local virtual environment (VE) in which the interaction is performed using the Leap Motion Controller (LMC) gesture sensor [10] and the Vybe haptic gaming pad [11]. The system, called GoodVybesConnect, allows the therapy input parameters to be individualized and calibrated according to the patient's characteristics. It also allows the execution of the therapy to be dependent on the therapist's hand movement in the VE, so that multimodal feedback (i.e., visual, audible, and vibrotactile feedback) is sent to patients in real time depending on the availability of the 


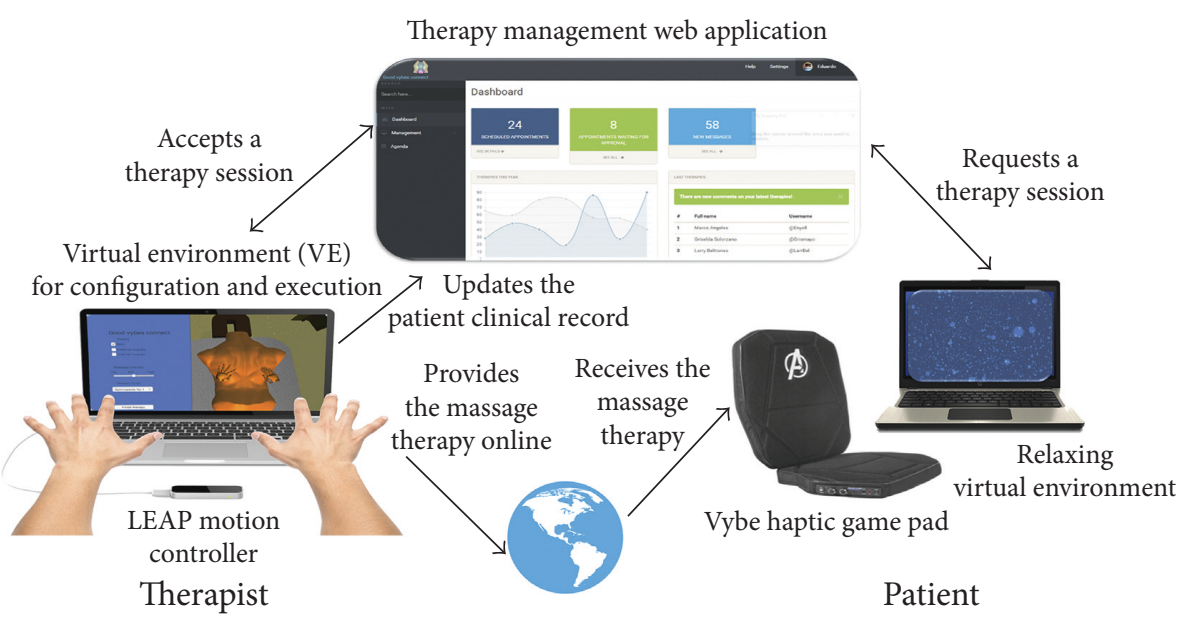

FIgure 1: The GoodVybesConnect architecture.

therapist. Otherwise, the patient might play at home a massage session previously scheduled by the specialist. Finally, therapy results are automatically updated in the patient's clinical record.

To assess the GoodVybesConnect current prototype and to identify some design insights that might help improving the current version of the system, we carried out two evaluations: the objective of the first evaluation was to identify the user experience of potential patients during a massage session, while the second one aimed at knowing the specialists' (i.e., therapists and psychologists) opinions regarding their usability perception of the application and their user experience while participating as patients and therapists.

\section{Related Work}

According to the literature, there are various forms of intervention for back treatment, including physical therapy, acupuncture, chiropractic treatment, and massage therapy, among others [12]. Massage therapy has been shown to affect both structure and function of the musculoskeletal system by promoting a relaxation response, decreasing muscle tension, and decreasing tonic muscle contractions [13-16]. In recent years, the integration of mechanical instruments to support the implementation of massage therapies such as massage chairs [5-7], automatic massage systems [6], or robotic systems [17-21] has benefited therapists and their patients.

The massage chair allows different massage techniques, for example, pressure point massage, roll stretch massage, and beat massage [5]. In some cases, depending on the patient's physiological variables being measured (e.g., mental condition, blood pressure, heart rate, and stress, among others), the specialist selects the massage technique that she/he considers the most appropriate [22-24]. There also exist automatic massage systems such as medical devices that combine basic principles of mechanical massage, thermotherapy, acupressure, infrared therapy, and moxibustion. For instance, the device described in [6] is capable of releasing modulated and controlled thermomechanical energy on the patient following a programmable and fully reproducible automatic treatment program selected by the operator. Another example of automatic massage systems is robotic systems that use massage patterns, for example, robots that provide maxillofacial massages [17], massage of the back $[20,21,25]$, or feet massage [18]. Nevertheless, the implementation of massage therapy with these mechanical instruments needs "hard-wired" programmed routines, local supervision of the therapist, and manual therapy management programs [26]. Moreover, these procedures also require the patient to be in the same place as the therapist, and they are mainly carried out in the traditional way using nonmechanical instruments $[3,12]$.

In comparison to the aforementioned references, our realtime haptic-enhanced telerehabilitation system [9] is innovative given that it (i) proposes a method to provide real-time back rehabilitation in a supervised and remote way, (ii) provides a web application for managing therapy programs, (iii) combines emerging tools to perform therapies, including a VE, the Vybe haptic gaming pad [11], and the LMC gesture sensor [10], and (iv) provides a secure way for patients to replay their massage according to the therapy program.

\section{Description of the System}

Figure 1 shows the architecture of the real-time haptic-enhanced telerehabilitation system. The GoodVybesConnect system was designed following therapists' recommendations, which were obtained through a contextual study performed in a rehabilitation center [9]. The system is composed of a webbased application (Figure 2) and a local VE (Figure 3).

The web-based application is a therapy administration system in which therapists can manage their therapy sessions' schedules and their patients as well as their corresponding clinical records. In addition, therapists can configure the therapy by adjusting the visual feedback, intensity, and kind of music, among others. The web application also shows the results of patients' past therapy sessions through tables and graphs (Figure 2). Moreover, patients are able to schedule a therapy session, view their therapy record, access past therapy sessions, and communicate via chat with their therapist. 


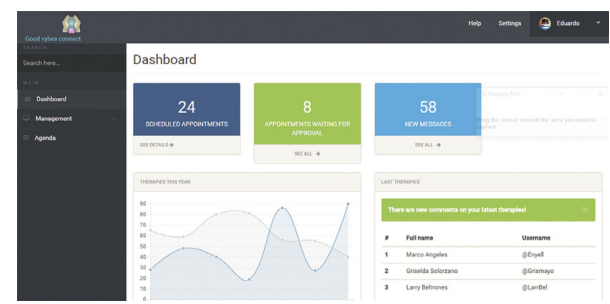

Figure 2: Therapy management web application. The therapist can accept, communicate, and update the patient's therapy record. In addition, the patient can request a therapy session, communicate with the therapist, and obtain his/her therapy files.

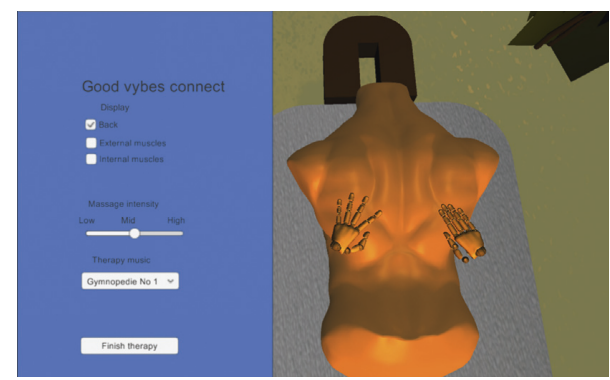

FIGURE 3: VE for the configuration and execution of back massage therapy.

The VE is a haptic-enhanced telerehabilitation system that was developed using Unity 5.3.4, the Vybe haptic gaming pad [11], and the Orion library for the LMC gesture sensor [10]. The main objective of the $\mathrm{VE}$ is to provide a real-time and remote back massage therapy to patients. The LMC gesture sensor is used as the input device, while the Vybe haptic gaming pad is used as the massage bed (output device) to execute therapy (Figure 1). The Vybe haptic gaming pad is a vibro-tactile grid display that has six voice-coil actuators located on the upper back and six DC motors located on the seat and lower back. The voice coils play smooth vibrations $(150-250 \mathrm{~Hz})$, while the DC motors play rumble-like effects [11].

Figure 3 shows the configuration screen in which the therapist chooses the virtual back that she/he wants to visualize (i.e., back muscles and surface or intermediate muscles), the intensity of the haptic feedback, and the music the patient will hear during therapy. The movements of the therapist's hands are detected with the LMC gesture sensor and transmitted online in real time to both the VE and the Vybe haptic gaming pad. All parameters can be changed online during execution of the therapy. After concluding the massage, the therapist stores the session and shares it through the web application with the patient so that she/he can reproduce his/her therapy again if indicated by his/her therapy program.

\section{User Experience Evaluation with Potential Patients}

4.1. Procedure. To obtain the participants' perception of usability and the participant's user experience when receiving a massage of the back with our system, we conducted

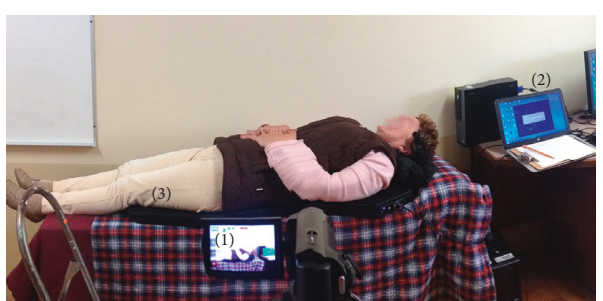

Figure 4: Participant during back massage therapy. Materials: (1) video camera, (2) computer running the VE for back massage therapy, and (3) haptic Vybe gaming pad used as massage bed.

a controlled usability test at the research laboratory of a local public university [9]. We used both questionnaires and controlled observation to evaluate whether the participant's user experience was pleasurable or not. That is, participants have a pleasant user experience when they feel relaxed and/or happy during the massage session, and they have an unpleasant user experience if they feel stressed, scared, or nervous.

The potential patients were 25 older adults (age: mean \pm SD: $63.68 \pm 7.98$ years, 11 males and 14 females), from whom $32 \%$ have received physical therapy due to various reasons (e.g., neck or spine problems, Parkinson's disease, or depression), $60 \%$ were under medical treatment, and $72 \%$ use the computer on a regular basis. All participants signed a consent form and agreed to be video-recorded during the execution of the back massage (Figure 4).

System Usability Scale (SUS) [27] and Technology Acceptance Model (TAM) [28] questionnaires (5-point Likert scale) were applied at the end of the study. During both the on-entry demographic questionnaire and the on-exit questionnaire, participants were asked about their emotional state at that moment, for example, if they were stressed, scared, nervous, relaxed, happy, or neutral. Besides, while answering the on-exit questionnaire, participants were asked for which of the aforementioned moods prevailed during the massage as well as their perception of the haptic feedback received through the Vybe haptic gaming pad. After that, two expert evaluators realized a controlled observation evaluation analyzing the video recordings to further verify the participants' moods.

4.2. Preliminary Results. From the on-exit questionnaire, we obtained the following results: $8 \%$ participants reported feeling neutral, 84\% relax, and 8\% happy. Further, during the analysis of the video recordings of the massage sessions, the expert evaluators identified that participants depicted the following moods: 92\% happy, 96\% relaxed, and 12\% nervous. However, participant's moods were not constant throughout the entire massage sessions, as they were observed during varying time periods and sometimes in a combined form, whereby the experts selected which mood predominated during the massage session, determining thus that the participants were observed: $92 \%$ relaxed and $8 \%$ nervous. Table 1 shows the moods reported by participants and observed predominantly in them by the experts. 
TABLE 1: The predominant moods reported by the subjects and observed by the experts (i.e., $1=$ stressed, $2=$ scared, $3=$ nervous, $4=$ neutral, $5=$ relaxed, and $6=$ happy).

\begin{tabular}{lccccccccccccccccccccccccccc}
\hline \multirow{2}{*}{ Result } & 1 & 2 & 3 & 4 & 5 & 6 & 7 & 8 & 9 & 10 & 11 & 12 & 13 & 14 & 15 & 16 & 17 & 18 & 19 & 20 & 21 & 22 & 23 & 24 & 25 \\
\hline Reported by the subject & 4 & 5 & 5 & 5 & 5 & 5 & 6 & 5 & 5 & 5 & 5 & 5 & 6 & 5 & 5 & 5 & 5 & 5 & 5 & 5 & 5 & 5 & 4 & 5 & 5 \\
Observed by evaluators & 5 & 5 & 5 & 5 & 3 & 3 & 5 & 5 & 5 & 5 & 5 & 5 & 5 & 5 & 5 & 5 & 5 & 5 & 5 & 5 & 5 & 5 & 4 & 5 & 5 \\
\hline
\end{tabular}

According to Table 1, it can be observed that there was a coincidence of $80 \%$ (20 cases) between how the participants self-perceived and what was observed by the experts. From these 20 instances, 19 cases coincide that they were relaxed and were thus observed by the experts (5-5), while on the remaining cases (that of participant P23), it is reported as having a neutral state during the massage, which was ratified by the observers (4-4). In the first case, it is concluded that the user experience was pleasant since it was declared so by the participants and ratified by the experts; in the second case, in the absence of enough evidence, the user experience of the participant was declared as not pleasant.

Additionally, the differences found in the rest of the participants include the case of participant P1 (4-5), the cases of participants P5 and P6 (5-3), and the cases of participants P7 and P13 (6-5). For the case of participant P1 (4-5), neutral self-perception versus observed relaxed, at the beginning of the session, the participant reported that, on one side of the device, she/he did not feel the same way as on the other; however, after a minute, the participant closed his/her eyes and he/she seemed relaxed during the rest of the session; therefore, the observers reported him/her as relaxed; however, it is likely that the problem of the device persisted and that the experience was not pleasant enough as to become relaxed or feel happy; so, the participant was selfperceived as neutral and we concluded that the participant did not have a pleasant experience.

Of the two participants in the tuple (5-3), self-perceived relaxed versus observed nervous, observers reported that, throughout the session, participants were observed unease; in the case of P5, she was observed during the whole session with her eyes open wide and blinking fast, and at no time, she seemed relaxed; with P6, something similar occurred, as he also constantly squeezed the lips and moved the fingers of his hands, so he was clearly nervous. This nervousness could be caused by the expectation of not knowing how the device worked. Neither P5 nor P6 had received some type of treatment or physical therapy in the past. In both cases, we concluded that the user experience was not pleasant.

Finally, two participants reported feeling happy during the session; however, according to the observation, they were perceived very relaxed (6-5). It can be said that they were enjoying the massage a lot, and although they were relaxed, they perceived themselves happier than relaxed. In both cases, it is considered then that their user experience was pleasant.

As conclusion, we have that $84 \%$ of the participants had a pleasant user experience when reporting themselves as relaxed or happy during the massage session and that, for the $16 \%$ remaining participants, there is no evidence that they had a pleasant user experience by presenting clear evidence of nervousness throughout the session or reporting a neutral emotional state.

\section{Evaluation with Specialists}

5.1. Procedure. The study was conducted with specialists in motor rehabilitation who participated in a system-extended usability test using the on-exit questionnaire inspection technique. The purpose of this evaluation was to obtain the specialists' perception about the usability of the system and their user experience while participating both as patients and therapists. In this way, we aim at assessing the usability of the current prototype and identifying some insights to improve the system.

The participants were 10 female specialists ( 7 therapists and 3 psychologists, mean age: $29.9 \pm 8.00$ years) who work in two different institutions $(7.25 \pm 5.95$ years average experience). The specialists provide motor or sensory therapy to various patients due to multiple etiologies (e.g., sensory disintegration, injury due to physical activity, lack of knowledge of the body, feeling exhausted, stress, and hypertension, among others). Seventy percent of them care for patients of all ages, while the rest provide care only for children with autism, social integration problems, and Down's syndrome, among others. Eighty percent of the specialists use common instruments to carry out the therapy, for example, swings, hammocks, textures, mattresses, and balls using their hands or knees, and the rest uses videos, songs, and stories to motivate patients. Finally, $100 \%$ consider that computer systems can be used as a support tool in therapy according to the diagnosis and treatment of the patient.

All participants signed a consent form and agreed to be video-taped during the execution of the evaluation. The evaluation was conducted at the work center of each specialist. The VE application was installed on a computer, while the Vybe haptic device was used as a massage bed (Figure 5).

In this evaluation, the specialists participated in two conditions: (a) $\mathrm{C} 1$, as patients and (b) $\mathrm{C} 2$, as therapists, so that they could have real experiences from both perspectives and enrich their perception of the system. Our hypotheses of the use of the system are as follows: (H1) the usability perception of the GoodVybesConnect system is higher for condition $\mathrm{C} 1$ than for condition $\mathrm{C} 2$ and (H2) the user experience of the participant in condition $\mathrm{C} 1$ is more pleasant than in condition $\mathrm{C} 2$.

The evaluation procedure consisted of a welcome message, initial explanation, and a questionnaire related to 


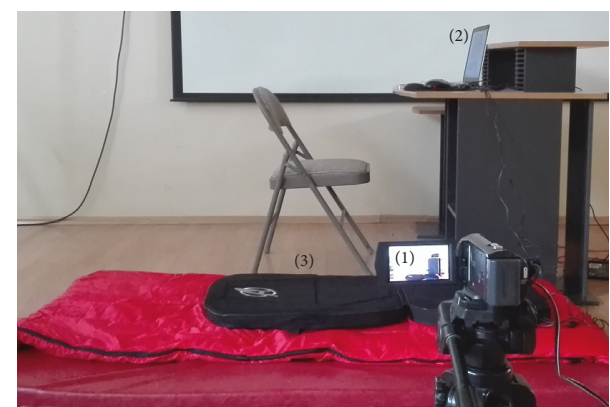

Figure 5: Example of the assessment scenario with specialists. Materials: (1) video camera, (2) computer running the VE for back massage therapy, and (3) haptic Vybe gaming pad used as massage bed.

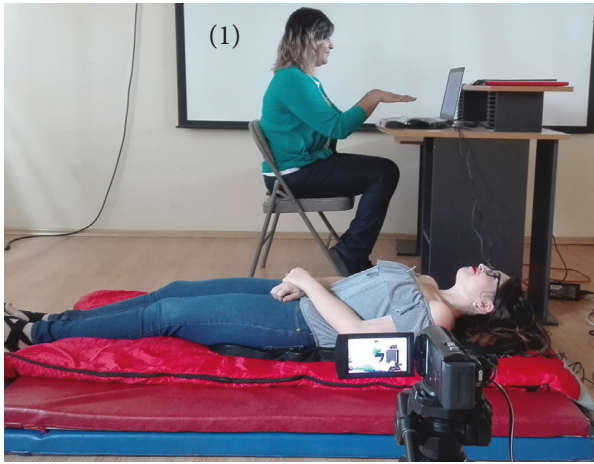

(a)

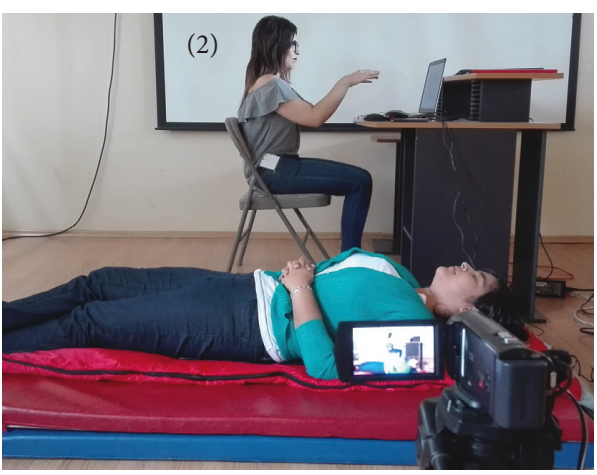

(b)

FIGURE 6: Specialists during back massage therapy. Scenario: (1) participant $\mathrm{P} 9$ as patient $(\mathrm{C} 1)$ and participant $\mathrm{P} 10$ as therapist (C2) and (2) participant P10 as patient (C1) and participant P9 as therapist (C2).

their demographic data. Then, in pairs, the specialists used the system for 4 minutes as a patient $(\mathrm{C} 1)$ and for 4 minutes as a therapist (C2) (Figure 6). Role assignment (i.e., therapist or patient) was randomly controlled to avoid bias. The intensity of the haptic feedback of the provided massage was also set randomly (i.e., $1 / 4,1 / 2,3 / 4$, and 1). All participants experienced the same auditory feedback during the massage.

System Usability Scale (SUS) and Technology Acceptance Model (TAM) questionnaires (5-point Likert scale) were applied at the end of the study. During both the on-
TABLE 2: Results of the evaluation in terms of the usability perception (H1).

\begin{tabular}{lcc}
\hline Hypothesis & \multicolumn{3}{c}{$\begin{array}{c}\text { Outcome } \\
\text { H1 (usability) }\end{array}$} \\
\hline Condition & $\mathrm{C} 1$ & $\mathrm{C} 2$ \\
M & 4.20 & 4.30 \\
IQR3 & 4.75 & 5.00 \\
IQR1 & 4.00 & 3.50 \\
\hline
\end{tabular}

C1: specialist as patient; C2: specialist as therapist; M: median; IQR: interquartile range.

TABle 3: Summary of Wilcoxon test on H1 hypothesis (critical value $T=3$ with $N=8$ ).

\begin{tabular}{lcc}
\hline Comparative & Statistical value & Significance of the difference \\
\hline H1 (usability, alpha $=0.05)$ & \\
C1 versus C2 & 14.5 & Not significant \\
\hline
\end{tabular}

$\mathrm{C} 1$ : specialist as patient; $\mathrm{C} 2$ : specialist as therapist.

entry demographic questionnaire and the on-exit questionnaire, participants were asked about their emotional state at that moment, for example, if they were stressed, scared, nervous, relaxed, happy, or neutral. Besides, while answering the on-exit questionnaire, participants were asked (using a 5-point Likert scale) which of the aforementioned moods prevailed during the massage (as therapist and as patient) and their perception of the haptic feedback received through the Vybe haptic gaming pad. Additionally, participants were asked about the system functions and haptic device characteristics and comments or suggestions about the system. Finally, a primary analysis of normality (using an input analyzer tool) indicated that the outcome variables were not normally distributed. Therefore, to analyze the hypotheses, a nonparametric statistical analysis was applied using the two-tailed nonparametric Wilcoxon test [29].

\section{Results}

6.1. Perception of Usability. Table 2 presents the summary of the participant's perception of usability regarding the system when using it as a patient (C1) and using it as a therapist (C2). As seen in Table 2, the usability perception is high in both conditions: as a patient (median 4.20/5) and as a therapist (median 4.30/5). In addition, the statistic test indicates that the differences in perception are not significant (see $\mathrm{H} 1$ in Table 3); therefore, $\mathrm{H} 1$ is rejected. Additionally, Figure 7 shows that although the differences in the usability perception of the GoodVybesConnect system were not significant, $70 \%$ of the specialists completely agree that the system is usable as a support tool in massage therapy, unlike their perspective as patients, where only $20 \%$ completely agree that the system is usable, and $60 \%$ of them agree.

All participants as patient (C1) stated that if they would have the system available, they would use it: $20 \%$ stated that they would use it once or twice a week, 30\% stated that they would use it three or four times a week, $30 \%$ stated that they 


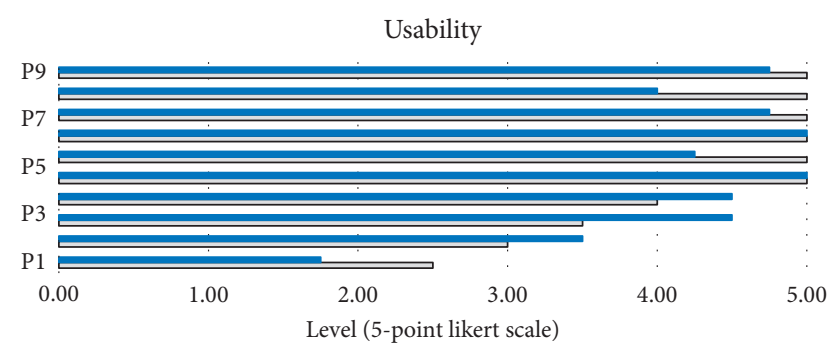

- C1: Specialist as patient $\quad \square \mathrm{C} 2$ : Specialist as therapist

FIGURE 7: Usability perception ( $\mathrm{H} 1)$. C1: specialist as patient; C2: specialist as therapist. Level: $1=$ totally disagree, 2 =disagreement, $3=$ neutral, $4=$ agree, and $5=$ completely agree .

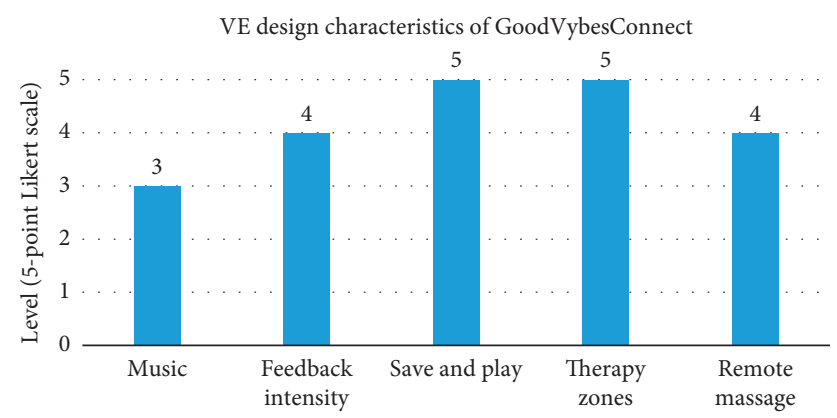

FIGURE 8: Importance level of design characteristics of the VE of GoodVybesConnect according to the specialist. Level: $1=$ totally disagree, $2=$ disagreement, $3=$ neutral, $4=$ agree, and $5=$ completely agree.

would use it 5 times a week, and $20 \%$ stated that they would use it 6 times a week or more. Regarding time of use, 50\% declared that they would use it for 10 minutes, $40 \%$ stated that they would use it for 15 minutes, and $10 \%$ stated that they would use it for 15 minutes or more.

Furthermore, $100 \%$ of the participants as therapist (C2) stated that if they would have the system available, they would use it: $10 \%$ stated that they would use it once or twice a week, $40 \%$ stated that they would use it three or four times a week, $40 \%$ stated that they would use it 5 times a week, and $10 \%$ stated that they would use it 6 times a week or more. Regarding time of use, $10 \%$ declared that they would use it for 5 minutes, $60 \%$ stated that they would use it for 10 minutes, and $30 \%$ stated that they would use it for 15 minutes or more.

In addition, regarding the use of the VE, the specialists indicated that they completely agree that the most important characteristics of the VE are saving and reproducing the therapy (median 5/5), as well as focusing on certain areas of therapy according to a therapy program $(5 / 5)$. The specialists also agreed that it is important that the massage can be performed remotely (median 4/5) and that the levels of intensity of the haptic feedback can be adjusted (median 4/5). Finally, changing the music in real time was the characteristic perceived as less important (median 3/5) (Figure 8).

Regarding the perception of the haptic feedback of the system according to the specialists (Figure 9), a good perception of the haptic feedback was obtained (median 4.18/5).

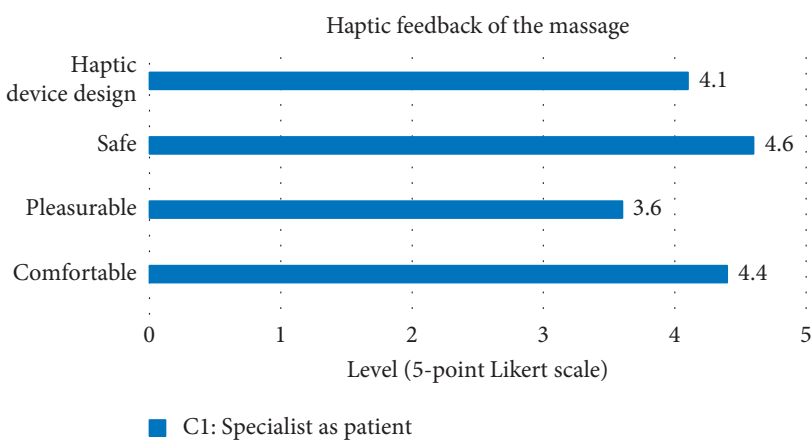

FIgURE 9: Massage haptic feedback perception (H2). C1: specialist as patient. Level: $1=$ totally disagree, $2=$ disagreement, $3=$ neutral, $4=$ agree, $5=$ completely agree.

Figure 9 shows the average scores perceived by the specialists. According to these results, the specialists felt first and foremost the safe feature (median 4.6/5); second, they liked the comfort provided by the mattress (median 4.4/5); third, they liked the design of the haptic device; and finally, the pleasurable feature had the lowest perception (median 3.6/5).

Finally, several specialists indicated that systems such as the GoodVybesConnect could be very useful to attend children who are unaware of their own body, who do not let themselves to be touched, or who have suffered any injuries, among others. In addition, in adult patients, it can be used to treat, for example, stress, anxiety, tension, muscle pain, or to relax patients who are prostrate due to illness.

6.2. User Experience. Table 4 shows the summary of the specialists' user experience upon receiving massage (condition C1) and providing massage (condition C2) (H2 hypothesis). In addition, Table 5 presents the result of the analysis in terms of the emotions presented by the specialists. As shown in Table 5, the results obtained show evidence that the differences between emotions during therapy on receiving $(\mathrm{C} 1)$ or providing $(\mathrm{C} 2)$ therapy are not significant, and therefore, $\mathrm{H} 2$ is discarded. However, on average, the user experience in condition $\mathrm{C} 1$ (receiving the massage) was slightly more pleasurable than in condition $\mathrm{C} 2$ (providing the massage).

Figure 10 shows the emotions of the specialists when using the GoodVybesConnect (H2) system. On the one hand, while feeling the mattress with haptic feedback and receiving the massage (C1), everyone denied feeling stressed (100\%), and most participants denied having felt fear $(90 \%)$ or being nervous $(80 \%)$. Likewise, it can be emphasized that most of them affirmed to have felt relaxed, (90\%) and all affirmed to have felt happy (100\%). On the other hand, when using the system and providing the massage (C2), most of them denied having felt stressed $(90 \%)$ or having felt fear $(80 \%)$; while some reported feeling nervous (30\%) or neutral (60\%). Finally, regarding feeling relaxed, $30 \%$ disagreed and $40 \%$ agreed or completely agreed; while most of them $(70 \%)$ indicated that they felt happy. 
TABLE 4: Results of the evaluation in terms of the emotions that the participants felt during the use of the system $(\mathrm{H} 2)$.

\begin{tabular}{lcc}
\hline Hypothesis & \multicolumn{2}{c}{ Outcome } \\
& \multicolumn{2}{c}{$\mathrm{H} 2$ (user experience) } \\
\hline Condition & $\mathrm{C} 1$ & $\mathrm{C} 2$ \\
M & 2.58 & 2.57 \\
IQR3 & 2.67 & 3.00 \\
IQR1 & 2.33 & 2.33 \\
\hline
\end{tabular}

C1: specialist as patient; $\mathrm{C} 2$ : specialist as therapist; M: median; IQR: interquartile range.

TABle 5: Summary of Wilcoxon test on H2 hypothesis (critical value $T=0$ with $N=6$ ).

\begin{tabular}{lcc}
\hline Comparative & Statistical value & Significance of the difference \\
\hline & H2 (user experience, alpha $=0.05)$ \\
C1 versus C2 & 7 & Not significant \\
\hline
\end{tabular}

C1: specialist as patient; C2: specialist as therapist.

\section{Discussion}

7.1. Perception of Usability. The main results suggest that the usability perception of the GoodVybesConnect system is high, both when using it as a patient (C1) (median 4.20/5) and as a therapist (C2) (median 4.30/5). Sixty percent of the specialists while using the system to provide the massage (condition C2) were in complete agreement (i.e., median 5/5) that the system is useful, easy to use, easy to learn, and with high intention to use (Figure 7). No significant differences were found regarding the usability perception of the GoodVybesConnect system in both conditions ( $\mathrm{C} 1$ and $\mathrm{C} 2$ ), since in both cases the perception of usefulness was high, and given that all the specialists indicated that they were willing to use the system once it becomes available.

In addition, the specialists evaluated the design features of the current system, indicating the following as the most important: (a) saving and reproducing a therapy session (median 5/5), (b) targeting therapy areas according to a program (Figure 8), (c) that the massage could be performed remotely (median 4/5), (d) that the intensity of the haptic feedback could be adjusted, and (e) that music could be changed in real time, with the latter being considered less important (median 3/5). As evidenced by these results, on average, the design characteristics of the system were considered important by the specialists.

The main findings in the perception of haptic feedback suggest that $50 \%$ of the specialists while receiving massage completely agree that the intensity is adequate (median 4.18/5) (Figure 9). However, the rest of the specialists indicated that it is necessary to increase and focus on the intensity of the massage feedback to generate more pleasant experiences of use. On the other hand, although the perception of the design of the device is adequate (median $4.3 / 5$ ), they indicate that it could have larger dimensions to cover other massage zones, including the neck, sides of the trunk, nape, and legs.

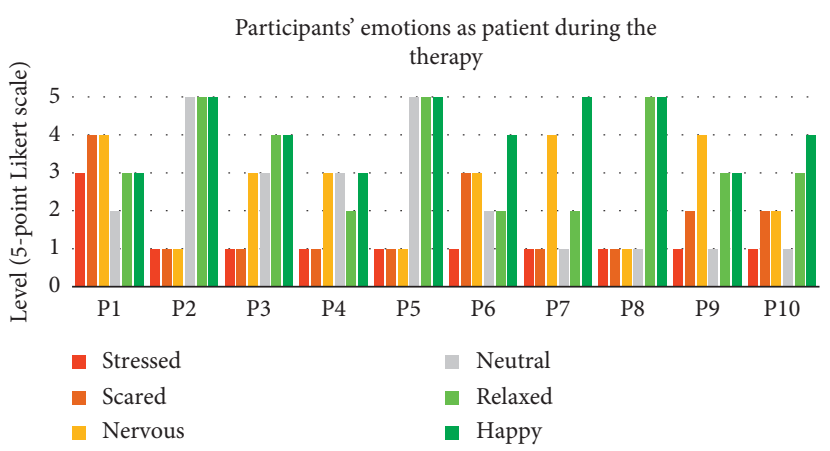

(a)

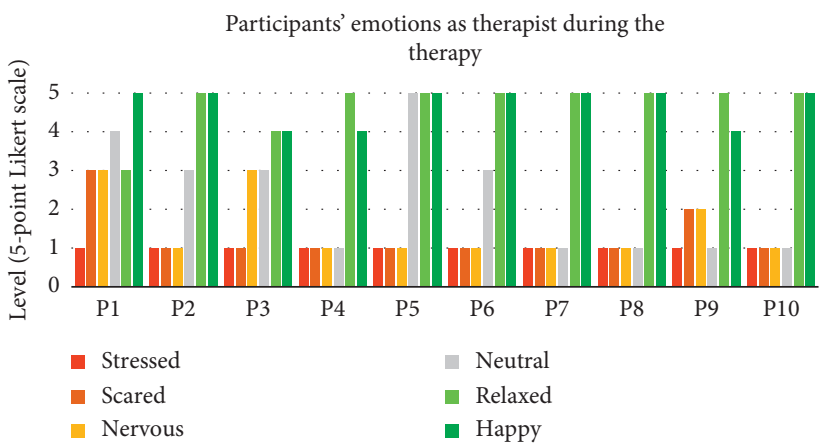

(b)

Figure 10: Emotions during therapy (H2). Left (C1: specialist as patient); right (C2: specialist as therapist). Level: $1=$ totally disagree, 2 = disagreement, $3=$ neutral, $4=$ agree, $5=$ completely agree.

7.2. User Experience. The analysis of the specialists' emotions upon receiving massage (condition $\mathrm{C} 1$ ) and on providing massage (condition $\mathrm{C} 2$ ) indicates that most specialists did not had a negative experience of use (i.e., stress or fear) and that their differences are not significant. However, P1 in his role of therapist (C2) expressed that he felt scared, which can be explained according to his final comments "I was afraid to do an incorrect movement when giving the massage" (P1). In addition, in the therapist role, the specialists P1, P7, and P9 answered that they were nervous when using the system, which could be explained based on some of their comments: "[it is necessary] to accompany the system with a user manual, or with a video tutorial, as not all therapists are used to interact with a virtual environment" (P7), "I found everything very good, it just made me nervous not to do it correctly" (P9) (Figure 10). This can also be explained by the fact that during the use of the GoodVybesConnect system, the specialists indicated feeling more relaxed when receiving the massage (88\%) than when giving the massage $(50 \%)$ (Figure 10). Additionally, in their perception while receiving the massage (C1), 90\% felt relaxed and 100\% felt happy. This higher percentage in positive experience of use might explain why at the end of the massage participants P2, P4, P5, P6, P7, P8, P9, and P10 asked to continue the massage; some of their comments were "why it ended" (P4, P5, P8, and P10), "I was falling asleep" (P2, P9, and P10), "it felt great, when will it be available?" (P6), and "where can I buy it?" (P5, P9, and P10). Finally, several of the specialists mentioned that they felt better when the intensity of the haptic feedback was 
greater and indicated that it would be advisable to increase it to improve its pleasurable feature.

According to the results of both evaluations, there is evidence that, on the one hand, the GoodVybesConnect system was well perceived by potential users and that it generated mainly pleasant user experiences. On the other hand, the specialists agreed on its high usability from the two perspectives ( $\mathrm{C} 1$ and $\mathrm{C} 2$ ), as well as on that it generated pleasant user experiences from the perspective of the patient. Specialists have also shared different proposals aiming at improving the design of the $\mathrm{VE}$ and of the massage cushion.

\section{Design Insights}

Based on the comments and suggestions made by specialists in rehabilitation, the following system design topics emerged:

(i) Adjustment of therapy strategies. The specialists suggested that it would be interesting if the patient could provide the massage in the absence of a therapist, or even have both the patient and the therapist able to select different preset therapy techniques to treat the patient. Some of the comments were: "it would be great if at any time there was the option of being able to direct your own [massage] when you do not have access to a therapist" (P6), "that the system could control itself to receive the massage" (P8), and "generate a prerecorded massage to use it with other patients" (P7).

(ii) Selection, delimitation, and adjustment of the massage zones. Specialists said that it is necessary to focus on the massage zones to avoid touching sensitive areas of the patient. They also suggested that it would be important to add the area of the head, neck, nape, trunk sides, and legs. They also recommend that the delimitation of the zones can be determined depending upon the corpulence of the person and their age, for example, child or adult. Some of the comments were: "inquire about the affected areas to give more emphasis" (P1), "isolate the stimulus of the pressure being exerted" (P2), "exert less intensity in the central zone (column)" (P7), and "consider the scapula" (P8).

(iii) Adequate calibration and size of the massage device. Specialists suggested that the haptic device be wider and longer. In addition, they mentioned that, once the device is larger, different dimensions can be configured to adapt the treatment according to the patient and add [supports for] the neck, sides of the trunk, shoulders, or legs. Some of the comments were: "I would like to add an attachment for the neck" (P2), "it is necessary to think about overweight people (obesity) because [in its current version] it may not cover their expectation for treatment" (P3), "the dimensions are suitable for standard people" (P3), "[it requires a] wider and longer mattress" (P4, P5), "maybe add [support] for sides and shoulders" (P6), "increase its dimensions [haptic device]" (P10), "the mattress could be wider for the benefit of larger people" (P10), and "[it] could cover the area of the legs" (P10).

(iv) Add temperature and increase the intensity of the haptic feedback of the massage. Specialists suggest that the stimulus for the patient was better at the higher intensity of the massage; in addition, they recommended adding temperature to strengthen the relaxation of the patients. Also, they considered that it is necessary to numerically quantify the intensity of the haptic feedback. Some of the comments were: "[it requires] intensity levels of feedback, consider them as a wider numeric scale rather than low, medium, and high" (P3), "higher feedback intensity of the mattress" (P1, P2, and P8), "I would like to add temperature to strengthen relaxation" (P2), "I felt better when the intensity of the massage was at the highest" (P3, P5, and P9), and "to relax more in the upper part of the back” (P8).

(v) Add haptic feedback to provide support to the movements of the therapist and improve the visual representation in the VE. The fear and nervousness emotions of the specialists while using the VE of GoodVybesConnect suggest that it is necessary to integrate the haptic feedback while providing the massage so that the therapist becomes aware of the intensity with which she/he is touching the patient. In addition, this intensity of the haptic feedback must correspond to the visual representation of the patient's virtual body in the VE. Some of the comments were: "I was afraid to do the movement incorrectly when giving the massage" (P1) and "I found everything very good, I just felt nervous of not doing it correctly" (P9).

\section{Conclusions}

A real-time haptic-enhanced telerehabilitation system for massage therapy was evaluated with potential users and specialists. In the evaluation with potential users, the experience of use was obtained, while with the specialists in addition to the experience of use, the perception of usability of the system was determined, along with some insights to improve its design.

To the best of our knowledge, GoodVybesConnect is the first system that allows therapists to perform back massage therapy in real time and remotely. Although we have evaluated the system with a small group sample, namely, 25 potential patients and 10 specialists, we would like to point out that, in the clinical literature domain, there are works where similar statistical tests are applied with a similar number of participants, for instance $[5,17,19]$. In addition, the HCI literature suggests that the inclusion of five experts during the evaluation stage makes it possible to detect 80 to $85 \%$ of the usability problems of a design proposal [30].

Furthermore, the results of both evaluations provide evidence of a high perception of usability, positive user experience (i.e., relaxation and joy), and good haptic feedback (i.e., safe, pleasurable, and comfortable) provided by 
the system. In addition, the comments suggest that the specialists perceived the current design features as important, and they generated new design insights focused primarily on improving massage feedback. Finally, as future work, it is necessary to further include the emerged design insights to enhance the current system. First, we will strengthen the design of the system to improve the experience of use and its clinical impact. Second, we are going to evaluate the proposed tool with more specialists (i.e., clinicians and therapists), and potential patients, to confirm the observed trends. Third, we will use the system for a longer period of time to better establish the scope and impact of these results.

\section{Conflicts of Interest}

The authors declare that there are no conflicts of interest.

\section{Acknowledgments}

The authors thank the therapists from the two rehabilitation centers at Ensenada, México, for participating in the study. This work was funded by Universidad Autónoma de Baja California under Grant 212 Proyectos de Servicio Social 2016 and CONACYT scholarship number 344565 provided to the first author.

\section{References}

[1] M. A. McCaskey, C. Schuster-Amft, B. Wirth, Z. Suica, and E. D. de Bruin, "Effects of proprioceptive exercises on pain and function in chronic neck- and low back pain rehabilitation: a systematic literature review," BMC Musculoskeletal Disorders, vol. 15, no. 1, p. 382, 2014.

[2] M. Rogante, M. Grigioni, D. Cordella, and C. Giacomozzi, "Ten years of telerehabilitation: a literature overview of technologies and clinical applications," NeuroRehabilitation, vol. 27, no. 4, pp. 287-304, 2010.

[3] D. C. Cherkin, K. J. Sherman, J. Kahn et al., "Effectiveness of focused structural massage and relaxation massage for chronic low back pain: protocol for a randomized controlled trial," Trials, vol. 10, no. 1, p. 96, 2009.

[4] M. Romanowski, J. Romanowska, and M. Grześkowiak, “A comparison of the effects of deep tissue massage and therapeutic massage on chronic low back pain," Studies in Health Technology and Informatics, vol. 176, pp. 411-414, 2012.

[5] D. F. Zullino, S. Krenz, E. Frésard, E. Cancela, and Y. Khazaal, "Local back massage with an automated massage chair: general muscle and psychophysiologic relaxing properties," Journal of Alternative and Complementary Medicine, vol. 11, no. 6, pp. 1103-1106, 2005.

[6] P. Buselli, R. Bosoni, G. Busè et al., "Effectiveness evaluation of an integrated automatic thermomechanic massage system $\left(\mathrm{SMATH}^{\circledR}\right.$ system) in non-specific sub-acute and chronic low back pain - a randomized double-blinded controlled trial, comparing SMATH therapy versus sham therapy: study protocol for a randomized controlled trial," Trials, vol. 12, p. 216, 2011.

[7] D. J. Engen, D. L. Wahner-Roedler, A. M. Nadolny et al., "The effect of chair massage on muscular discomfort in cardiac sonographers: a pilot study," BMC Complementary and $\mathrm{Al}$ ternative Medicine, vol. 10, no. 1, p. 50, 2010.
[8] S. McEwen, "Social work in health care when conventional meets complementary: nonspecific back pain and massage therapy," Health and Social Work, vol. 40, no. 1, pp. 19-25, 2015.

[9] C. Ramírez-Fernández, E. García-Canseco, A. L. Morán et al., "GoodVybesConnect: a real-time haptic enhanced tele-rehabilitation system for massage therapy," in Proceedings of the 10th International Conference, UCAmI 2016, pp. 487-496, San Bartolomé de Tirajana, Gran Canaria, Spain, November-December 2016.

[10] A. H. Smeragliuolo, N. J. Hill, L. Disla, and D. Putrino, "Validation of the Leap Motion Controller using markered motion capture technology," Journal of Biomechanics, vol. 49, no. 9, pp. 1742-1750, 2016.

[11] Disney, "Disney research," 2015, https://www.disneyresearch. $\mathrm{com} /$.

[12] W. L. Chen, G. J. Liu, S. H. Yeh, M. C. Chiang, M. Y. Fu, and Y. K. Hsieh, "Effect of back massage intervention on anxiety, comfort, and physiologic responses in patients with congestive heart failure," Journal of Alternative and Complementary Medicine, vol. 19, no. 5, pp. 464-470, 2013.

[13] K. G. Davis and S. E. Kotowski, "Preliminary evidence of the short-term effectiveness of alternative treatments for low back pain," Technology and Health Care, vol. 13, no. 6, pp. 453-462, 2005.

[14] J. Baranowsky, P. Klose, F. Musial, W. Haeuser, G. Dobos, and J. Langhorst, "Qualitative systemic review of randomized controlled trials on complementary and alternative medicine treatments in fibromyalgia," Rheumatology International, vol. 30, no. 1, pp. 1-21, 2009.

[15] L. Gaskell, S. Enright, and S. Tyson, "The effects of a back rehabilitation programme for patients with chronic low back pain," Journal of Evaluation in Clinical Practice, vol. 13, no. 5, pp. 795-800, 2007.

[16] Y. Noto, M. Kudo, and K. Hirota, "Back massage therapy promotes psychological relaxation and an increase in salivary chromogranin A release," Journal of Anesthesia, vol. 24, no. 6, pp. 955-958, 2010.

[17] H. Ishii, H. Koga, Y. Obokawa, J. Solis, A. Takanishi, and A. Katsumata, "Path generator control system and virtual compliance calculator for maxillofacial massage robots," International Journal of Computer Assisted Radiology and Surgery, vol. 5, no. 1, pp. 77-84, 2010.

[18] J. Wang and Y. Li, "Massaging human feet by a redundant manipulator equipped with a tactile sensor," in Proceedings of the 2010 IEEE/ASME International Conference, pp. 7-12, Montreal, Canada, July 2010.

[19] R. C. Luo, C. W. Hsu, and S. Y. Chen, "Electroencephalogram signal analysis as basis for effective evaluation of robotic therapeutic massage," in Proceedings of the 2016 IEEE/RSJ International Conference on Intelligent Robots and Systems (IROS), pp. 2940-2945, Daejeon, Republic of Korea, October 2016.

[20] H. Gao, S. Lu, G. Tian, and J. Tan, "Vision-integrated physiotherapy service robot using cooperating two arms," International Journal on Smart Sensing and Intelligent Systems, vol. 7, no. 3, pp. 1024-1043, 2014.

[21] C. S. Meera, P. S. Sairam, and M. K. Gupta, "Path planning and motion control for a 3 DOF massaging robot," in Proceedings of the 2016 International Conference on Robotics and Automation for Humanitarian Applications (RAHA), pp. 1-6, Amritapuri, Kerala, India, December 2016.

[22] T. Teramae, D. Kushida, F. Takemori, and A. Kitamura, "Estimation of comfortable/uncomfortable feeling based on 
EEG by using NN and k-means algorithm for massage chair," IEEJ Transactions on Electronics, Information and Systems, vol. 131, no. 1, pp. 102-108, 2011.

[23] H. Jaafar, A. Fariz, S. A. Ahmad, and N. A. M. Yunus, "Intelligent massage chair based on blood pressure and heart rate," in Proceedings of the 2012 IEEE EMBS Conference on Biomedical Engineering and Sciences (IECBES), pp. 514-518, Langkawi, Malaysia, December 2012.

[24] J. Kim, K. B. Lee, S. Lee, H. Yang, and S. G. Hong, "A novel stress measurement system with handhold electrodes in massage chairs," in Proceedings of the 2016 International Conference on Information and Communication Technology Convergence (ICTC), pp. 859-863, Jeju Island, Republic of Korea, October 2016.

[25] R. C. Luo and C. C. Chang, "Electromyographic signal integrated robot hand control for massage therapy applications," in Proceedings of IEEE/RSJ 2010 International Conference on Intelligent Robots and Systems (IROS 2010), pp. 3881-3886, Taipei, Taiwan, 2010.

[26] C. Clar, A. Tsertsvadze, R. Court, G. Hundt, A. Clarke, and P. Sutcliffe, "Clinical effectiveness of manual therapy for the management of musculoskeletal and non-musculoskeletal conditions: systematic review and update of UK evidence report," Chiropractic and Manual Therapies, vol. 22, no. 1, p. 12, 2014.

[27] J. Brooke, "SUS-a quick and dirty usability scale," Usability Evaluation in Industry, vol. 189, no. 194, pp. 4-7, 1996.

[28] F. Davis, "Perceived usefulness, perceived ease of use, and user acceptance of information technology," MIS Quarterly, vol. 13, no. 3, pp. 319-340, 1989.

[29] J. H. Zar, Biostatistical Analysis, Prentice-Hall, Upper Saddle River, NJ, USA, 2010.

[30] J. Nielsen and R. Molich, "Heuristic evaluation of user interfaces," in Proceedings of the SIGCHI Conference on Human Factors in Computing Systems, pp. 249-256, Seattle, WA, USA, March 1990. 

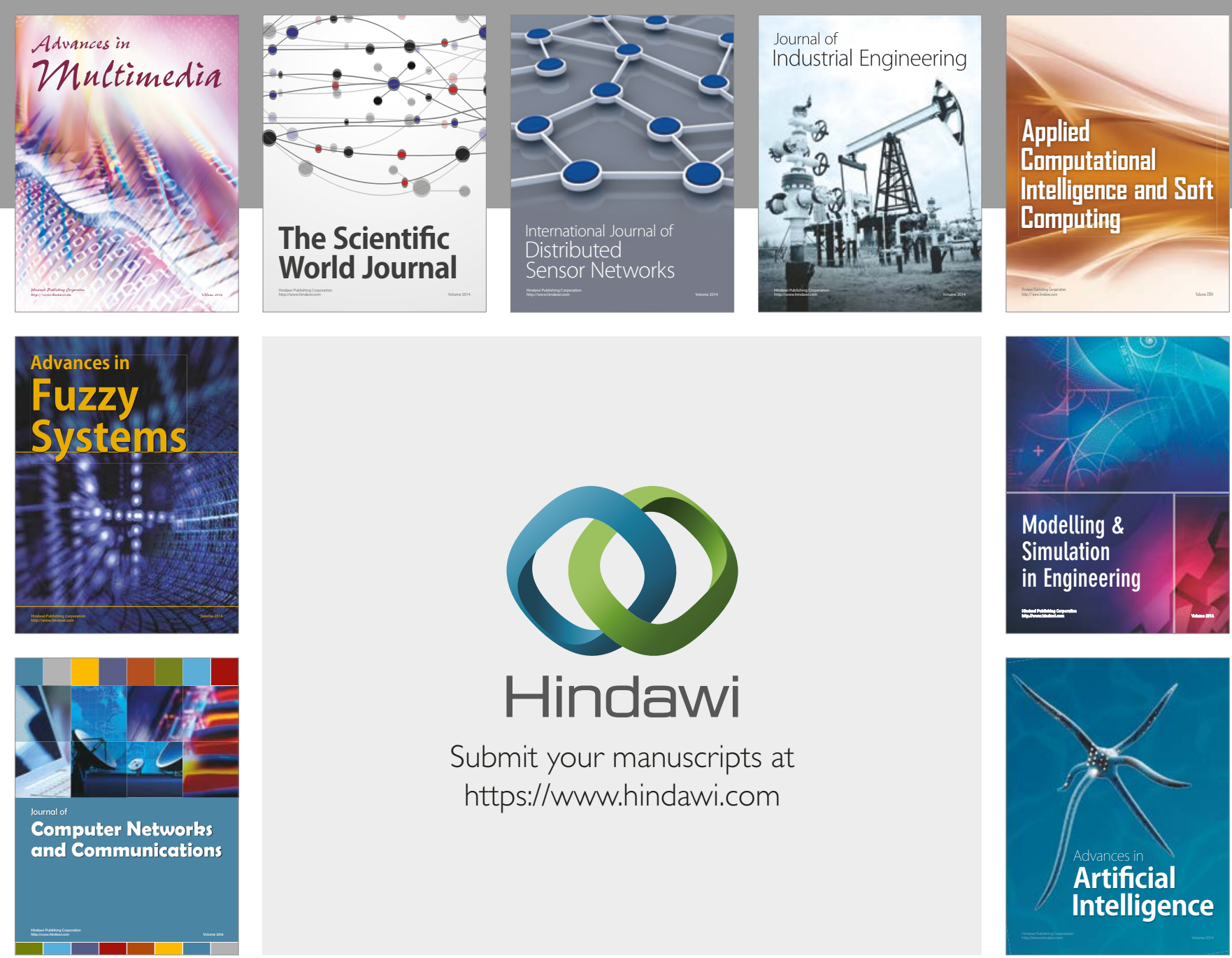

\section{Hindawi}

Submit your manuscripts at

https://www.hindawi.com
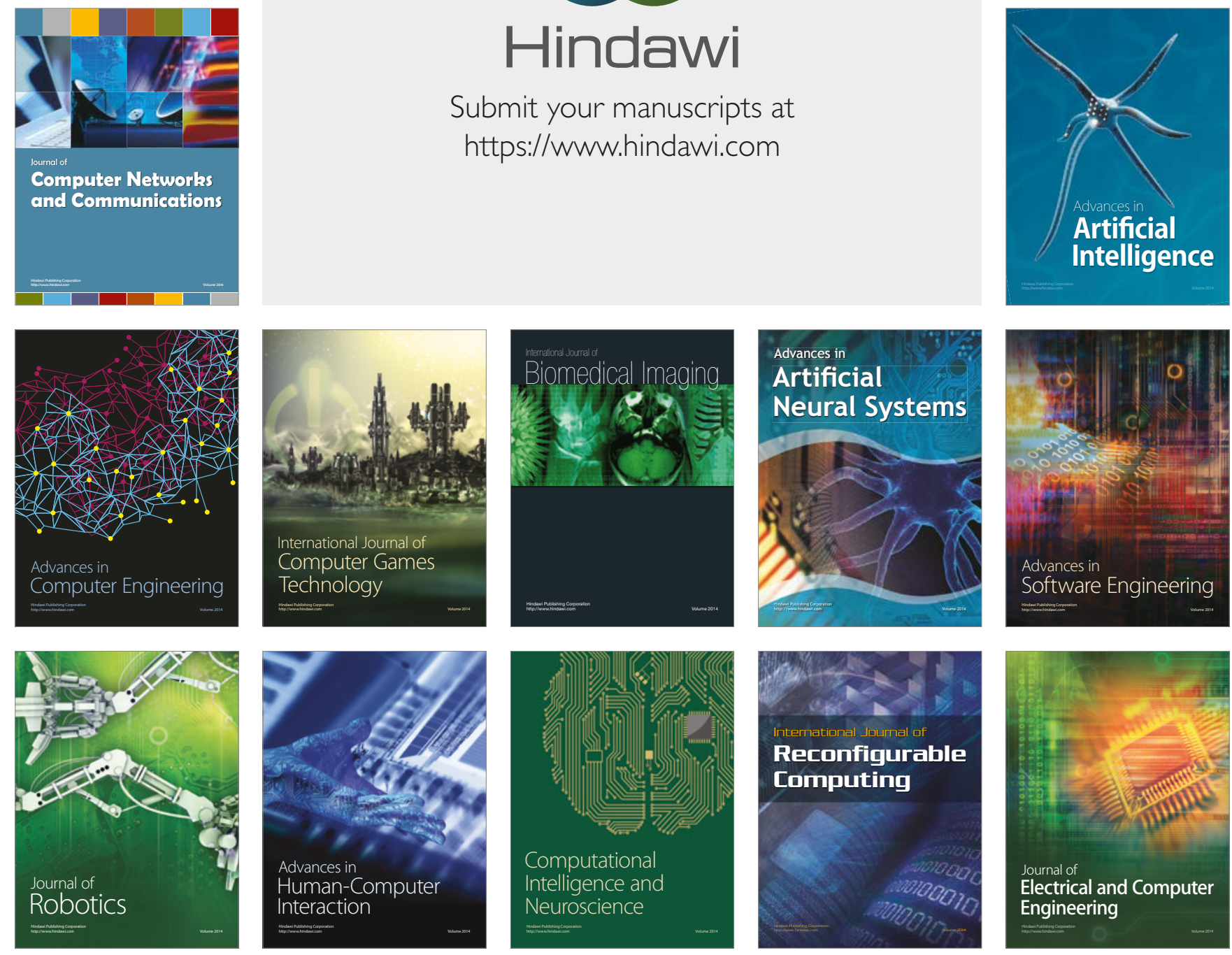